\title{
The QCD Dirac Operator Spectrum and Finite-Volume Scaling
}

\author{
P.H. Damgaard ${ }^{\mathrm{a} *}$ \\ aThe Niels Bohr Institute, Blegdamsvej 17, DK-2100 Copenhagen, Denmark \\ Random matrix theory and chiral Lagrangians offer a convenient tool for the exact calculation of microscopic \\ spectral correlators of the Dirac operator in a well-defined finite-volume scaling regime.
}

\section{INTRODUCTION}

The succesful analytical computations of the so-called microscopic QCD Dirac operator spectrum from random matrix theory [1 3 , have raised an important question. Why can random matrix theory, which seems to be very far from the quantum field theory of QCD, apparently be used to compute spectral properties of the Dirac operator? Within the last year this issue has been much clarified, with important consequences also for lattice gauge theory. The results obtained in random matrix theory are now seen to be exact predictions of QCD itself, in an appropriate finite-volume scaling regime. This is defined by [4] $1 / \Lambda_{Q C D}<<L<<1 / m_{\pi}$, where $L=V^{1 / 4}$ is the linear extent of the four-volume $V$. In this region one is probing finite-volume effects of QCD to the extreme. Only static modes of the lowest hadronic excitations contribute to the euclidean partition function of QCD, and if chiral symmetry is spontaneously broken according to $S U\left(N_{f}\right) \times S U\left(N_{f}\right) \rightarrow S U\left(N_{f}\right)$, it reads [4]

$\mathcal{Z}_{\nu}=\int_{U\left(N_{f}\right)} d U(\operatorname{det} U)^{\nu} e^{V \Sigma \operatorname{ReTr}\left[\mathcal{M} U^{\dagger}\right]}$

in a sector of fixed topological charge $\nu$. Here $\Sigma=\langle\bar{\psi} \psi\rangle$ is the quark condensate, and $\mathcal{M}$ is the quark mass matrix. In this context it is most useful to view $\mathcal{Z}_{\nu}$ as the generating function of $\bar{\psi} \psi$ (because this is how one sees that it contains information about the Dirac operator spectrum). It depends on the masses only in the particular combination $\mu=m V \Sigma$. This makes $\mathcal{Z}_{\nu}$ a finitesize scaling function.

\footnotetext{
*Work supported by EU TMR Grant no. ERBFMRXCT97-0122.
}

\subsection{The double-microscopic limit}

Eigenvalues $\lambda_{n}$ of the Dirac operator are determined by $i \not D \phi_{n}=\lambda_{n} \phi_{n}$. By comparing (1) with the original path integral (after integrating out the fermions),

$\mathcal{Z}_{\nu}=\prod_{f} m_{f}^{\nu} \int[d A]_{\nu} \prod_{f, n}\left(\lambda_{n}^{2}+m_{f}^{2}\right) e^{-S[A]}$,

one infers immediately that also the non-zero eigenvalues $\lambda$ in this finite-volume limit can only enter in the combination $\zeta=\lambda V \Sigma$. It is thus a double-microscopic limit $\lambda, m \rightarrow 0$ as $V \rightarrow \infty$ with $\mu$ and $\zeta$ fixed that will correspond to this finitevolume scaling regime [1]:5,3]. Remarkably, in this limit random matrix theory can be used to give am entirely equivalent description of the partition function (1). The relevant random matrix theory is given by [4

$\tilde{\mathcal{Z}}_{\nu}=\int d W \prod_{f} \operatorname{det}\left(M+m_{f}\right) e^{-\frac{N}{2} \operatorname{tr} V\left(M^{2}\right)}$

where

$M=\left(\begin{array}{cc}0 & i W^{\dagger} \\ i W & 0\end{array}\right)$

and $W$ is a rectangular complex matrix of size $N \times(N+|\nu|)$. This matrix ensemble, which is the one relevant for $\mathrm{QCD}$, is known as the chUE. The choice of ensemble, and the determinantal structure in (3), is fixed by the symmetry properties of the Dirac operator [6], while nothing a priori fixes the matrix potential $V\left(M^{2}\right)$. For simplicity, denote also the eigenvalues of $M$ by $\lambda$. In ref. [i] it was shown for the particular case of a Gaussian random matrix potential that in the 
double-microscopic limit the two partition functions agree (up to an irrelevant mass-independent constant):

$\mathcal{Z}_{\nu}\left(\mu_{1}, \ldots, \mu_{N_{f}}\right)=\tilde{\mathcal{Z}}_{\nu}\left(\mu_{1}, \ldots, \mu_{N_{f}}\right)$.

The required correspondences between the two formulations are $\Sigma \leftrightarrow \pi \rho(0)$ and $V \leftrightarrow 2 N$, where $\rho(\lambda)$ is the density of the random matrix eigenvalues. One particular consequence of a series of universality theorems (see ref. [7] and the first of ref. [3]) is that this equality holds universally, i.e. independently of the choice of random matrix model potential [8]. The only essential requirement is, as one could expect, that $\rho(0) \neq 0$. This means that masses and eigenvalues can be rescaled to common values. 2

The universal equivalence (5) is the root from which all exact relations between random matrix theory and QCD in the finite-volume scaling regime $1 / \Lambda_{Q C D}<<L<<1 / m_{\pi}$ follow. Because there are long-established techniques for computing spectral correlation functions in random matrix theory, this equivalence is not just interesting from a conceptual point of view, but is of great practical value.

\section{DIRAC SPECTRA AND PARTITION FUNCTIONS}

One particularly simple way of computing the microscopic spectral correlators of the Dirac operator eigenvalues is by relations to partition functions with additional quark species. In random matrix theory based on the chUE ensemble there exists a convenient shortcut for the calculation of spectral correlation functions which is based on the kernel $K_{N}\left(z, z^{\prime} ; m_{1}, \ldots, m_{N_{f}}\right)$. From this kernel alone one can derive all spectral correlation functions in the limit $N \rightarrow \infty$ :

$$
\begin{aligned}
& \rho\left(\lambda_{1}, \ldots, \lambda_{n} ; m_{1}, \ldots, m_{N_{f}}\right) \\
& \quad=\operatorname{det}_{a, b} K\left(\lambda_{a}, \lambda_{b} ; m_{1}, \ldots, m_{N_{f}}\right) .
\end{aligned}
$$

This relation is exact, and not restricted to the double-microscopic limit. When comparing the

\footnotetext{
${ }^{2}$ It is tempting to consider the case of $\rho(0) \rightarrow 0$ in random matrix language as the limit of chiral symmetry restauration, but there is presently no obvious classification of quantum field theories that match the new random matrix theory universality classes with $\rho(0)=0$.
}

explicit expression for the kernel with the eigenvalue description of the random matrix partition function (3) itself, one finds a very simple relation involving the ratio of two partition functions, one corresponding to having two additional quark species of imaginary mass! In the doublemicroscopic limit this relation simplifies even further, and if we now make use of the equivalence (5), we can express this kernel entirely in terms of finite-volume partition functions in the scaling region $1 / \Lambda_{Q C D}<<L<<1 / m_{\pi}$ [10]:

$$
\begin{aligned}
& K_{S}\left(\zeta, \zeta^{\prime} ; \mu_{1}, \ldots, \mu_{N_{f}}\right)=\prod_{f} \sqrt{\left(\zeta^{2}+\mu_{f}^{2}\right)\left(\zeta^{\prime 2}+\mu_{f}^{2}\right)} \\
& \quad \times \sqrt{\zeta \zeta^{\prime}} \frac{\mathcal{Z}_{\nu}\left(\mu_{1}, \ldots, \mu_{N_{f}}, i \zeta, i \zeta^{\prime}\right)}{\mathcal{Z}_{\nu}\left(\mu_{1}, \ldots, \mu_{N_{f}}\right)},
\end{aligned}
$$

up to an overall normalization constant that can be fixed by matching with the spectral density at the origin. Eq. (7) is a master formula from which all microscopic spectral properties of the QCD Dirac operator can be derived. In particular, the microscopic spectral density itself is obtained by taking the coincident limit $\zeta=\zeta^{\prime}$ :

$$
\begin{aligned}
& \rho_{S}\left(\zeta ; \mu_{1}, \ldots, \mu_{N_{f}}\right)=|\zeta| \prod_{f}\left(\zeta^{2}+\mu_{f}^{2}\right) \\
& \times \frac{\mathcal{Z}_{\nu}\left(\mu_{1}, \ldots, \mu_{N_{f}}, i \zeta, i \zeta\right)}{\mathcal{Z}_{\nu}\left(\mu_{1}, \ldots, \mu_{N_{f}}\right)}
\end{aligned}
$$

again up to an overall constant, which is easily fixed [10]. Although it involves a theory with two more quarks, one can also view it as the expectation value of a complicated operator in the original theory with just $N_{f}$ quarks.

It turns out that also the distribution $P_{S}\left(\zeta ; \mu_{1}, \ldots, \mu_{N_{f}}\right)$ of just the smallest Dirac operator eigenvalue can be expressed in terms of the finite-volume partition function. In the sector of topological charge $\nu=0$ it reads [1]

$$
\begin{aligned}
& P_{S}\left(\zeta ; \mu_{1}, \ldots, \mu_{N_{f}}\right)= \\
& -\frac{\partial}{\partial \zeta}\left[e^{-\zeta^{2} / 4} \frac{\mathcal{Z}_{0}\left(\sqrt{\mu_{1}^{2}+\zeta^{2}}, \ldots, \sqrt{\mu_{N_{f}}^{2}+\zeta^{2}}\right)}{\mathcal{Z}_{0}\left(\mu_{1}, \ldots, \mu_{N_{f}}\right)}\right]
\end{aligned}
$$

and similar expressions can be derived for any $\nu$.

The expressions (8) and (9) for the microscopic spectral density and smallest eigenvalue distribu- 
tions are the most convenient quantities to measure in lattice gauge theory. Their analytical expressions, using the explicit form of the finitevolume QCD partition function [12], agree precisely with results obtained earlier based directly on random matrix theory computations [2] 3]. Since the analytical results are known for arbitrary masses (keeping the condition $1 / \Lambda_{Q C D}<<$ $\left.L<<1 / m_{\pi}\right)$, they can in principle be measured by Monte Carlo techniques without difficulty even with dynamical fermions. Existing Monte Carlo meaurements 13] for QCD in 4 dimensions are based on staggered fermions and an $S U(2)$ gauge group, for which predictions actually are slightly different. Very recently the expression (8) has been derived directly from the chiral Lagrangian framework, without relying on the relation to random matrix theory at all [14].

\subsection{QCD in 3 dimensions}

One can also entertain the idea of spontaneous breaking of "chiral" symmetries in 3-dimensional QCD. Because fermions are described by twospinors, and $\gamma$-matrices thus can be taken as the Pauli matrices (and minus these matrices, a different representation of the Clifford algebra here), there is no room for an analogue of $\gamma_{5}$. However, by pairing up quark fields one can reformulate the theory in terms of four-spinors. Two hidden chiral symmetries (based on $\gamma_{4}$ and $\gamma_{5}$ rotations) then become manifest. For an even number of flavors it is conceivable that flavor symmetry, in the guise of chiral symmetry, can break spontaneously according to $U\left(N_{f}\right) \rightarrow U\left(N_{f} / 2\right) \times$ $U\left(N_{f} / 2\right)$, a case that has been analyzed in the random matrix framework in [15]. It recently been studied on the lattice in a quenched simulation, and indeed a condensate was observed at zero temperature, disappearing at the deconfinement phase transition [16]. In the broken phase one can study the microscopic Dirac operator spectrum, which turns out to be somewhat different from the 4-dimensional counterpart.

The case of quenched 3-dimensional QCD is the only known case for which the prediction for the microscopic spectral density is exactly flat. New Monte Carlo results on lattices of sizes ranging from $6^{3}$ to $14^{3}$, show fairly good agreement with this prediction [16]. Getting a flat microscopic distribution is in fact quite non-trivial, since generically the spectral density reflects the underlying peaks corresponding to the averaging over individual peaks of the smallest eigenvalues. It will of course be more interesting to see the result of an analogous simulation with dynamical fermions, for which the analytical prediction is a highly non-trivial oscillatory function [15].

\section{REFERENCES}

1. E.V. Shuryak and J.J.M. Verbaarschot, Nucl. Phys. A560 (1993) 306.

2. J.J.M. Verbaarschot and I. Zahed, Phys. Rev. Lett. 70 (1993) 3852.

3. P.H. Damgaard and S.M. Nishigaki, Nucl. Phys. B518 (1998) 495; T. Wilke, T. Guhr and T. Wettig, Phys. Rev. D57 (1998) 6486.

4. H. Leutwyler and A. Smilga, Phys. Rev. D46 (1992) 5607.

5. J. Jurkiewicz, M.A. Nowak and I. Zahed, Nucl. Phys. B478 (1996) 605; E: B513 (1998) 759 .

6. J.J.M. Verbaarschot, Phys. Rev. Lett. 72 (1994) 2531.

7. G. Akemann, P.H. Damgaard, U. Magnea and S. Nishigaki, Nucl. Phys. B487 (1997) 721.

8. P.H. Damgaard, hep-th/9807026.

9. G. Akemann, P.H. Damgaard, U. Magnea and S. Nishigaki, Nucl. Phys. B519 (1998) 682.

10. P.H. Damgaard, Phys. Lett. B424 (1998) 322; G. Akemann and P.H. Damgaard, hepth/9801133; hep-th/9802174.

11. S.M. Nishigaki, P.H. Damgaard and T. Wettig, hep-th/9803007.

12. A.D. Jackson, M.K. Şener and J.J.M. Verbaarschot, Phys. Lett. B387 (1996) 355.

13. M.E. Berbenni-Bitsch et al., Phys. Rev. Lett. 80 (1998) 1146; M.E. Berbenni-Bitsch, S. Meyer and T. Wettig, hep-lat/9804030.

14. J.C. Osborn, D. Toublan and J.J.M. Verbaarschot, hep-th/9806110.

15. I. Zahed and J.J.M. Verbaarschot, Phys. Rev. Lett. 73 (1994) 2288; P.H. Damgaard and S.M Nishigaki, Phys. Rev. D57 (1998) 5299.

16. P.H. Damgaard, U.M. Heller, A. Krasnitz and T. Madsen, hep-lat/9803012. 